\title{
CMEARTICLE \\ ST-segment changes with exercise stress
}

Yoke Ching $\underline{\operatorname{Lim}}^{1}$, MBChB, MRCP, Swee-Guan ${\underline{T e 0^{2}}}^{2}$ MRCP, FACC, Kian-Keong $\underline{P o h}^{1,3}$, FRCP, FACC

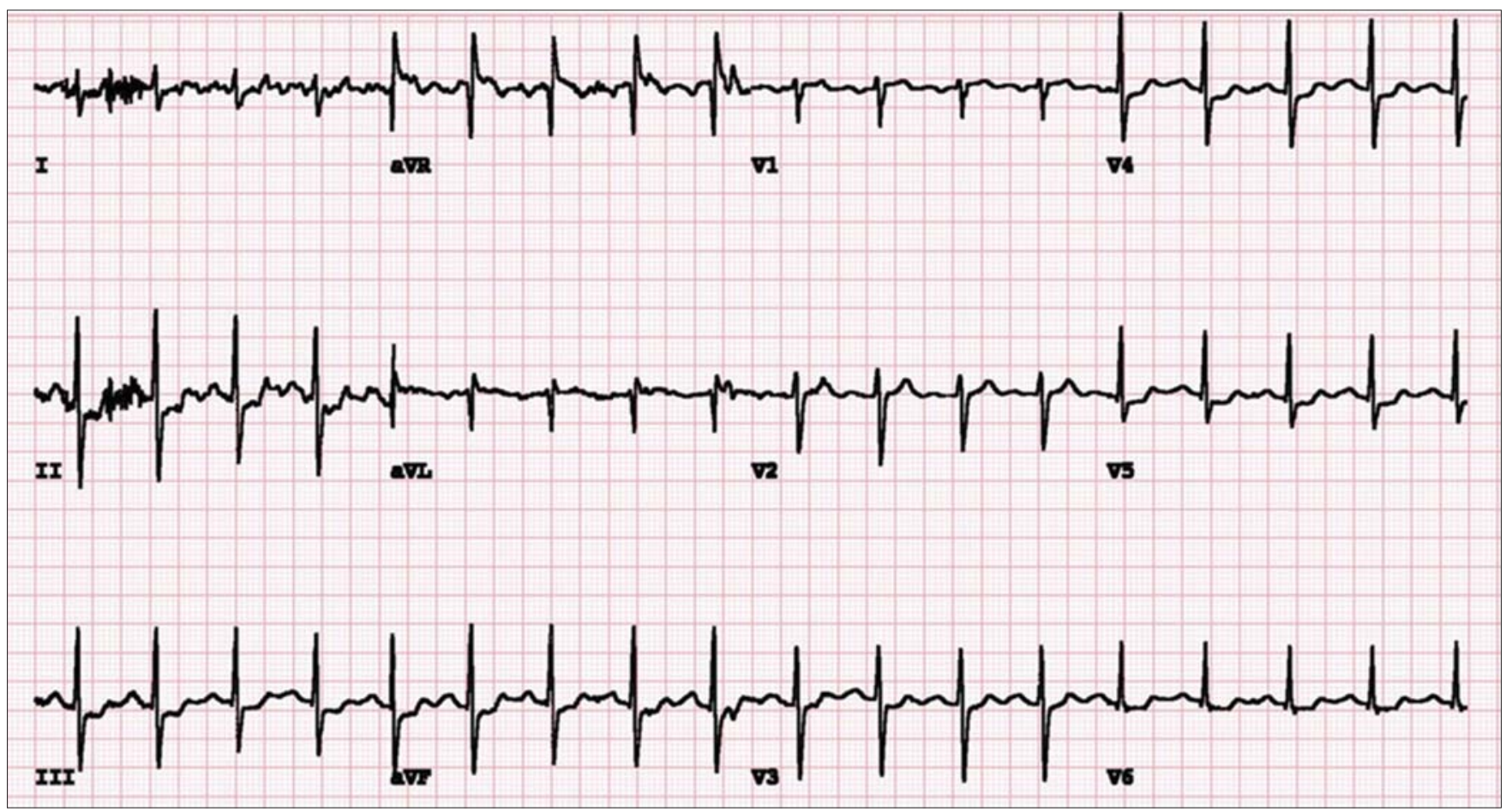

Fig. 1 ECG at peak stress during treadmill ECG stress test.

\section{CASE 1}

\section{CLINICAL PRESENTATION}

A 62-year-old woman presented with atypical angina for the past three months. Her past medical history includes hyperlipidaemia. Electrocardiography (ECG) performed at rest shows normal sinus rhythm. The patient underwent treadmill ECG stress test, and her ECG at highest heart rate during the treadmill stress test is shown in Fig. 1. What are the ECG abnormalities? What is the diagnosis?

\section{ECG STRESS TEST INTERPRETATION}

The test was terminated at Stage 2 of the Bruce protocol with seven Metabolic Equivalents of Tasks (METs) of work due to reports of chest pain and breathlessness. The patient could not achieve the target heart rate of 135 beats per minute (bpm). ECG performed at her highest heart rate (Fig. 1) shows sinus tachycardia at a rate of $112 \mathrm{bpm}$, with a 1-mm horizontal ST-segment depression at $80 \mathrm{~ms}$ after the J-point, in the inferolateral leads (II, III, aVF and V4-6), which is suggestive of ischaemia due to obstructive coronary artery disease (CAD). In addition, the patient also had a hypertensive response to exercise with a blood pressure of up to $206 / 96 \mathrm{mmHg}$.

\section{CLINICAL COURSE}

In view of her symptoms and abnormal treadmill ECG stress test, the patient underwent coronary angiography, which showed severe left main and triple vessel disease. She had $90 \%$ ostial left main stenosis, severe diffuse distal disease in the left anterior descending (LAD) and circumflex arteries, as well as $90 \%$ ostial right coronary artery (RCA) stenosis with $70 \%$ stenosis in the posterior descending artery (Fig. 2). The patient was referred to a cardiothoracic surgeon, and coronary artery bypass graft surgery was performed.

${ }^{1}$ Department of Cardiology, National University Heart Centre, National University Health System, ${ }^{2}$ Raffles Heart Centre, Raffles Hospital, ${ }^{3}$ Yong Loo Lin School of Medicine, National University of Singapore, Singapore

Correspondence: A/Prof Poh Kian Keong, Department of Cardiology, National University Heart Centre, 1E Kent Ridge Road, NUHS Tower Block Level 9, Singapore 119228. kian_keong_poh@nuhs.edu.sg 

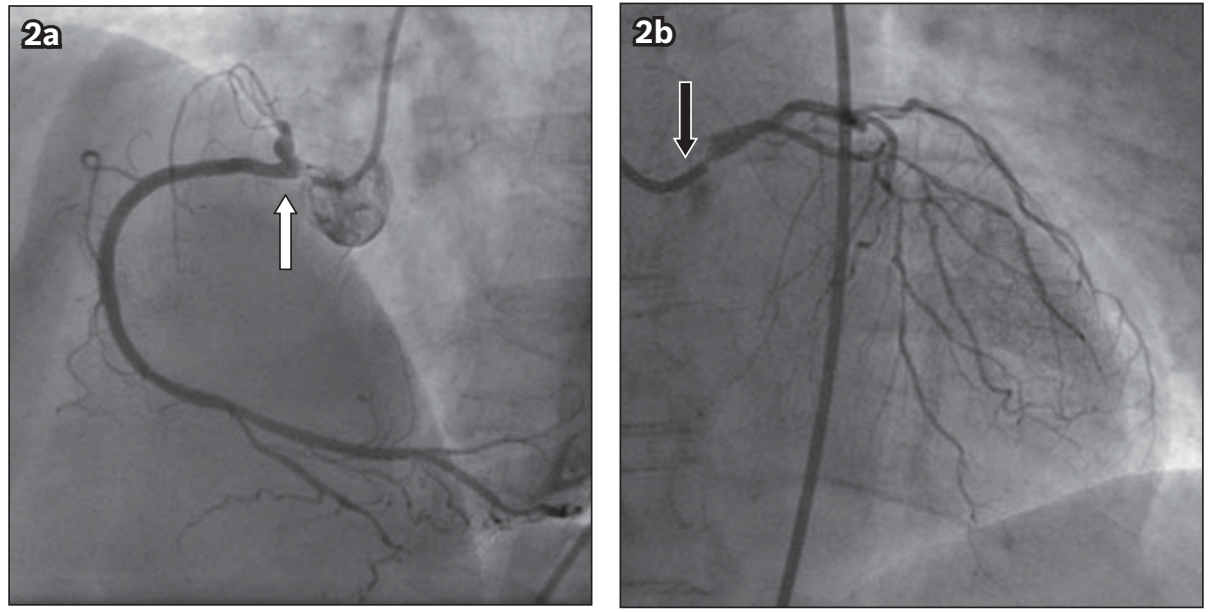

Fig. 2 Coronary angiograms show (a) 90\% stenosis in the ostium of the right coronary artery (white arrow) in the left anterior oblique view; and (b) 90\% stenosis in the ostium of the left main coronary artery (black arrow) with diffuse disease in the left anterior descending and left circumflex artery in the right anterior oblique cranial view.

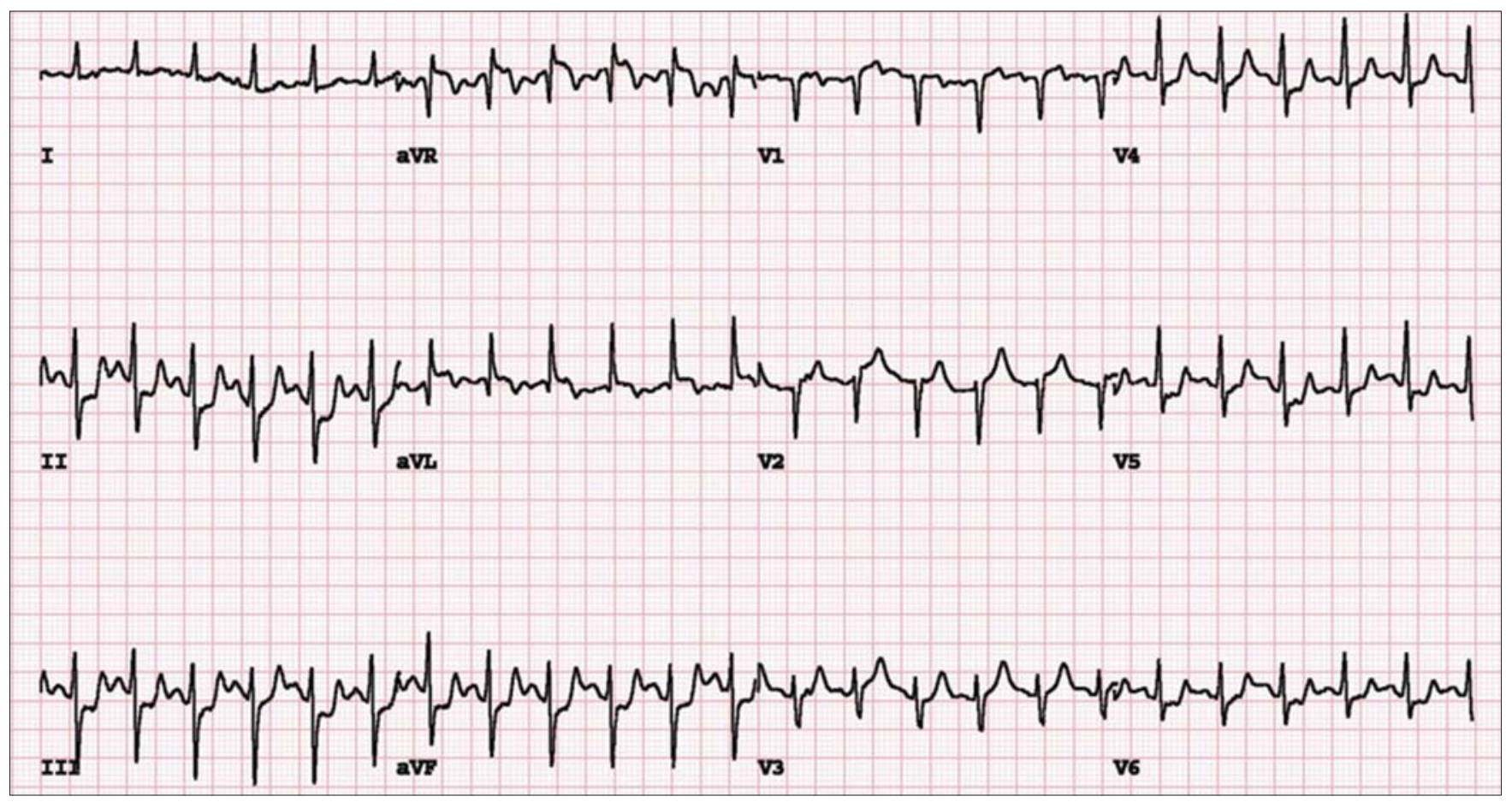

Fig. 3 ECG at peak stress during treadmill ECG stress test.

\section{CASE 2}

\section{CLINICAL PRESENTATION}

A 51-year-old man was referred for treadmill ECG stress test due to breathlessness on exertion. He had a previous anterior ST-elevation myocardial infarction in the year 2005 and primary percutaneous intervention to his proximal LAD coronary artery. The patient's medical history also included diabetes mellitus and dyslipidaemia. ECG done at rest shows Q waves in the anterior lead V1-2, consistent with a previous anterior myocardial infarction. The patient underwent treadmill ECG stress test. The ECG in Fig. 3 was taken at his maximal heart rate during stress. What are the ECG abnormalities?

\section{ECG STRESS TEST INTERPRETATION}

The patient achieved Stage 4 of the Bruce protocol, exercising for a total of nine minutes 46 seconds with 11.2 METs of work. ECG at peak stress showed sinus tachycardia at a rate of $146 \mathrm{bpm}$. There were new horizontal and up-sloping ST-segment depressions in leads II, III, aVF and V4-6, which were suggestive of myocardial ischaemia. In addition, there was an isolated 1-mm ST-segment elevation in lead $\mathrm{aVL}$. The ST segments returned to baseline at five minutes of recovery.

\section{CLINICAL COURSE}

The patient subsequently underwent coronary angiography, which showed total occlusion in the ostial LAD artery, with further $90 \%$ stenosis in the left posterior descending artery (LPDA) (Fig. 4). He underwent successful coronary angioplasty with stenting of the ostial to proximal LAD arteries and balloon angioplasty of the LPDA. The patient's effort tolerance improved after angioplasty. 


\section{CASE 3}

\section{CLINICAL PRESENTATION}

A 61-year-old man presented with a one-week history of angina. He had a history of hypertension and dyslipidaemia. The patient underwent myocardial perfusion treadmill ECG stress test. ECG at rest showed normal sinus rhythm. Fig. 5 shows the patient's ECG immediately after achieving target heart rate during stress. What are the ECG abnormalities?

\section{ECG STRESS TEST INTERPRETATION}

ECG performed immediately after achieving target heart rate (Fig. 5) showed sinus tachycardia with a heart rate of $100 \mathrm{bpm}$. The test was stopped, as the patient developed breathlessness at Stage 3 with a peak heart rate of $111 \mathrm{bpm}$, at 10.2 METs of work. ST-segment elevations of up to $2 \mathrm{~mm}$ were seen in the inferior leads II, III and aVF. In addition, there were also horizontal and down-sloping ST-segment depressions in leads I, aVL and V4-6.

\section{CLINICAL COURSE}

The patient's myocardial perfusion images showed a reversible perfusion defect in the mid-to-basal inferior wall, which is suspicious of obstructive CAD in the RCA territory. His coronary angiogram showed total occlusion of the proximal RCA, with Rentrop Grade 2 collaterals from the left coronary arteries to the distal RCA (Fig. 6). The patient underwent angioplasty with stenting to the RCA.

\section{DISCUSSION}

\section{Treadmill ECG stress test}

In 2014, ischaemic heart disease (IHD) was the third leading cause of death in Singapore, accounting for $16 \%$ of total deaths. ${ }^{(1)}$ There is a need for early identification and improved treatment of IHD. The treadmill ECG stress test is commonly used as a screening test to identify myocardial ischaemia. During exercise, limitation

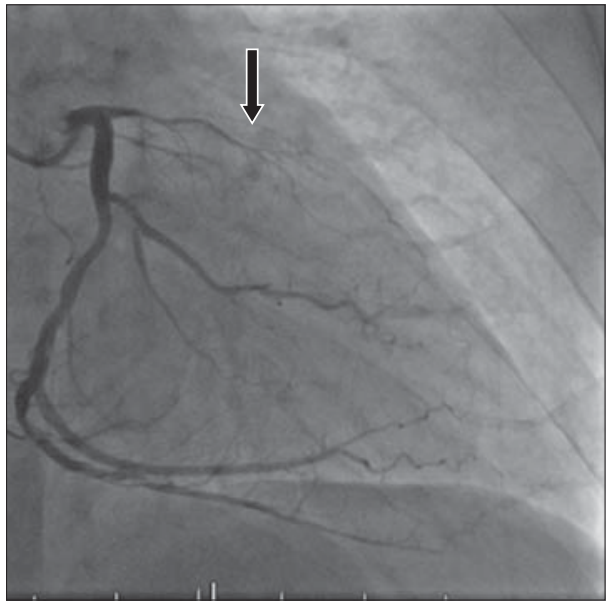

Fig. 4 Coronary angiogram shows total occlusion of the ostial left anterior descending artery in the right anterior oblique caudal view. The occluded left anterior descending stent is also seen (arrow).

of coronary blood flow due to obstructive CAD may lead to myocardial ischaemia and its subsequent ECG changes. This is the basis of detecting significant CAD on the treadmill ECG stress test. In addition, the treadmill ECG stress test is also frequently used to assess for chronotropic response to exercise, exerciseinduced arrhythmia and evaluation of symptoms during exercise, as well as the individual's exercise capacity. The sensitivity of the treadmill ECG stress test in diagnosing obstructive CAD was approximately $50 \%$, with a specificity of approximately $90 \%$. $^{(2)}$

\section{Indications for the treadmill ECG stress test}

The American Heart Association previously published a guideline recommending stress tests for adult patients with intermediate pretest probability of CAD. ${ }^{(3)}$ Table I provides a guide on the pretest probability of CAD for individuals based on age, gender and presenting symptoms.

The Ministry of Health, Singapore, published its own guidelines on screening for cardiovascular disease. ${ }^{(4)}$ In addition

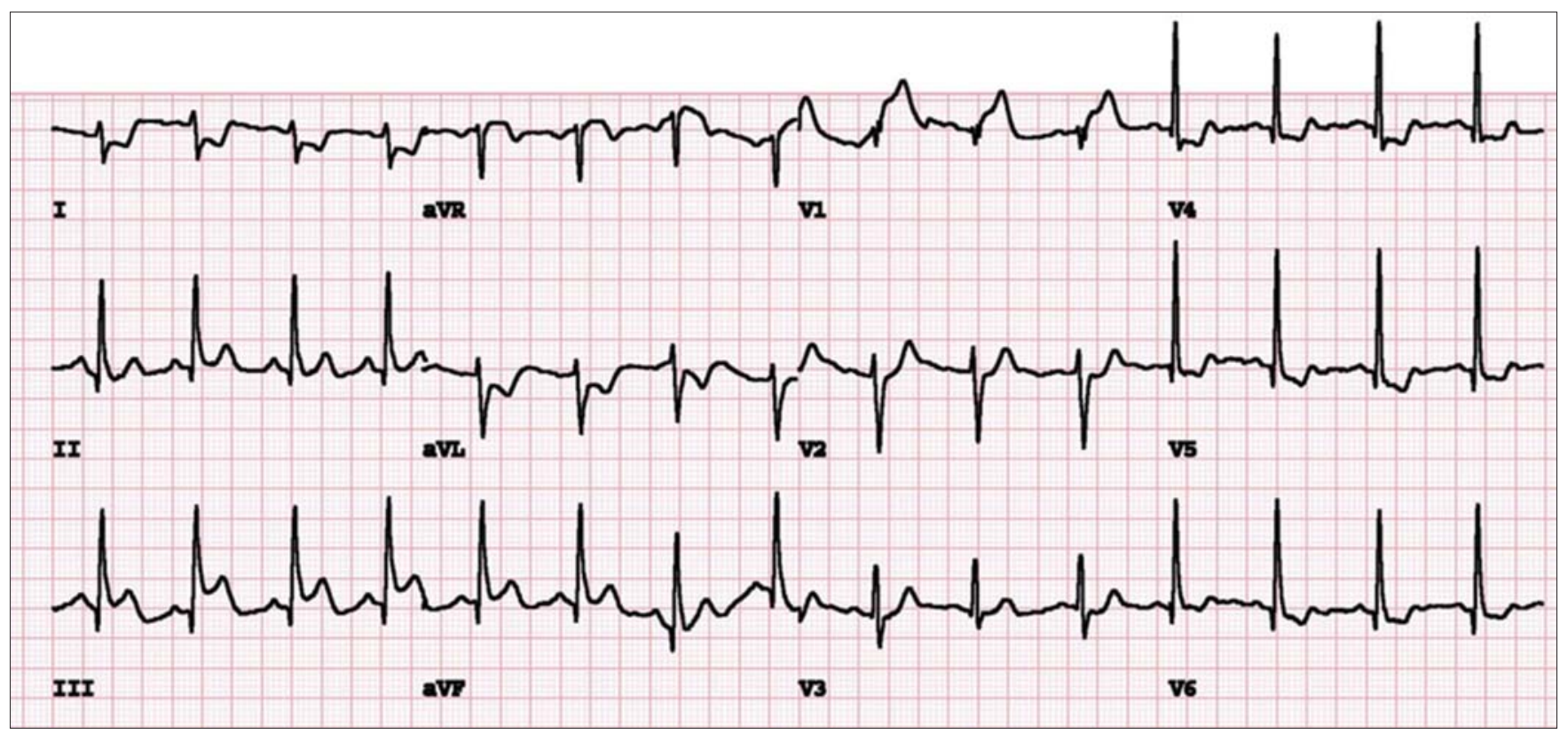

Fig. 5 ECG done immediately after peak stress. 

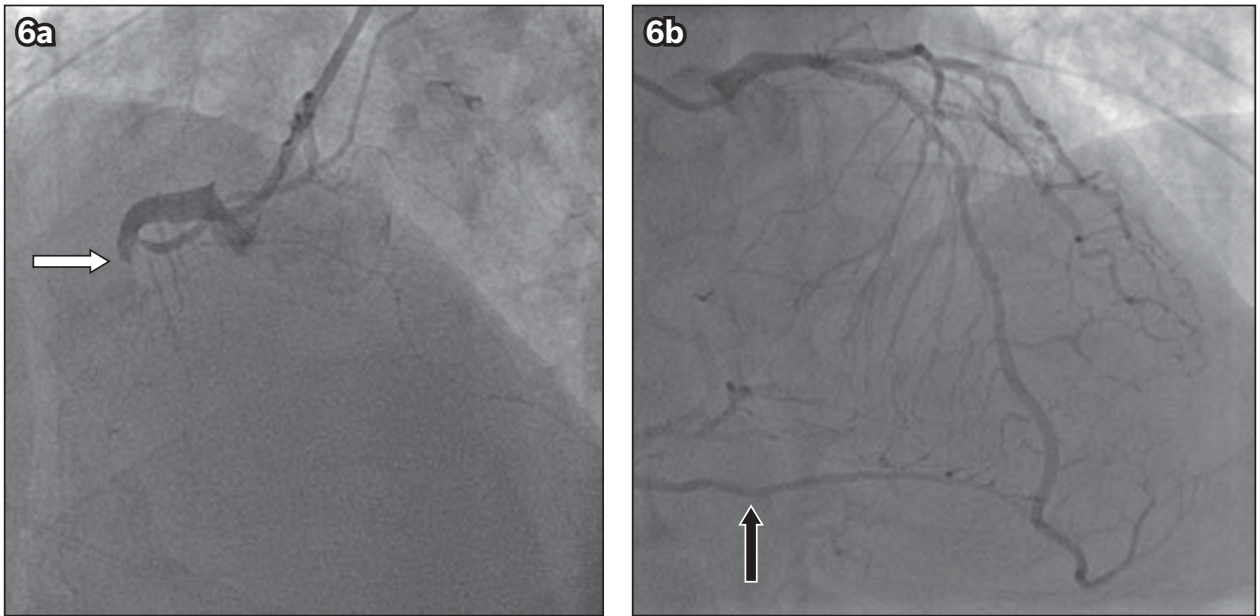

Fig. 6 Coronary angiogram shows (a) 100\% occlusion of the proximal right coronary artery (white arrow) in the left anterior oblique cranial view; and (b) collateral from the left coronary arteries (black arrow) in the left anterior oblique cranial view.

Table I. Pretest probability of coronary artery disease based on age, gender and presenting symptoms. ${ }^{(3)}$

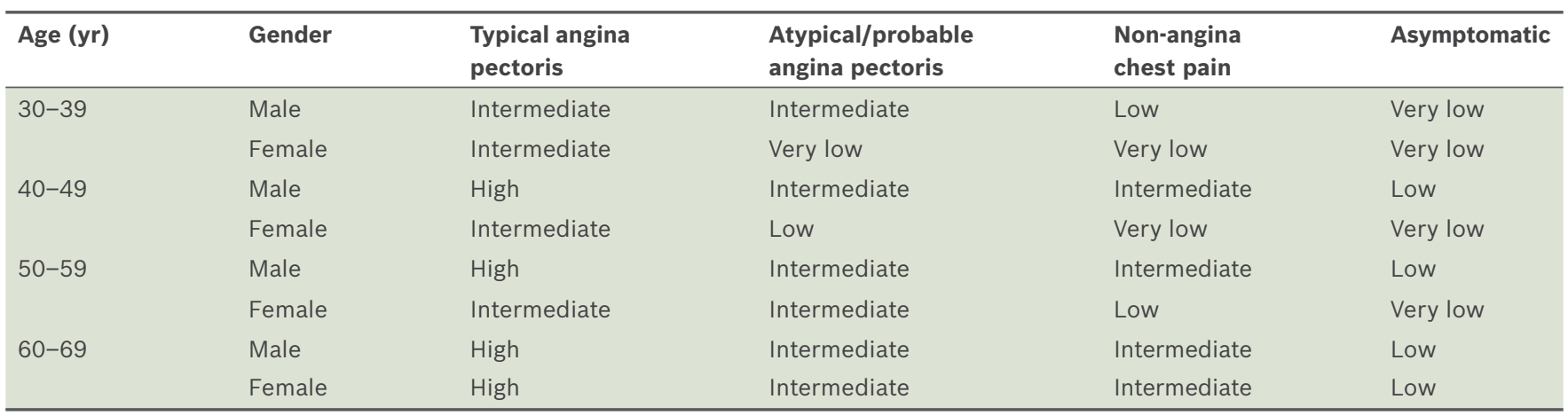

Categories are very low: < 5\%; low: $\geq 5 \%$ to < 10\%; intermediate: $10 \%-90 \%$; and high: $>90 \%$. Typical angina is defined as retrosternal chest pain provoked by exertion or emotional stress and relieved by rest or nitroglycerin. Atypical angina lacks one of these three characteristics.

to evaluating symptomatic patients with intermediate pretest probability, the guidelines also recommended the treadmill ECG stress test for the detection of CAD in the following groups: (a) asymptomatic individuals with multiple risk factors; (b) asymptomatic men above 45 years of age and women above 55 years of age who are planning to start vigorous exercise; (c) individuals in occupations in which impairment may affect public safety (e.g. pilots); (d) individuals at high risk of coronary artery disease due to comorbidities; and (e) patients with diabetes mellitus who are planning to start vigorous exercise.

Patients whose baseline ECG shows more than 1-mm ST-depression or less than 1-mm ST-depression, and who are taking digoxin or fulfil the ECG criteria for left ventricular hypertrophy, electronically paced ventricular rhythm or complete left bundle branch block were considered not suitable for evaluation using the treadmill ECG stress test alone, as it would be difficult to interpret any changes in the ST segment during the test.

\section{Performing the treadmill ECG stress test}

The Bruce protocol is the most commonly used exercise treadmill stress protocol. It consists of a total of seven stages, with a gradual increment in the speed and gradient of the treadmill. Each stage lasts three minutes to allow the patient to acclimatise to the specific speed and gradient before advancing to the next stage.
Exercise intensity during the treadmill ECG stress test is calculated in METs, which reflect the amount of oxygen consumed per minute. 1 MET is equal to $3.5 \mathrm{~mL} / \mathrm{min} / \mathrm{kg}$ of oxygen used. Watching television, for example, requires $1 \mathrm{MET}$, while brisk walking requires $4 \mathrm{METs}$.

Prior to starting the Bruce protocol, ECG may be performed with the patient hyperventilating in the standing position to assess for any ST-segment changes caused by hyperventilation. The MET value increases as the patient advances to higher stages of the Bruce protocol. Table II shows a standard Bruce protocol indicating the speed, gradient and MET values of each stage. Continuous ECG monitoring is carried out throughout the test and into the recovery period to assess for any changes in the ST segments, as well as for arrhythmia. The test is often stopped before completing Stage 7, when the patient achieves the target heart rate or is symptomatic, or when the test is positive. Other indications for termination are listed in Table III.

\section{Contraindications for exercise stress test}

As with any investigation, it is important to ensure the safety of the patient undergoing the test, since the treadmill ECG stress test could be harmful to the patient in certain situations. Each laboratory has its own protocol regarding the safety and suitability of the patients undergoing such tests. Table IV shows the main contraindications for a treadmill ECG stress test. 


\section{Heart rate response}

During exercise, the physiological increase in heart rate is due to a decrease in vagal tone followed by an increase in sympathetic tone. This, in turn, leads to increased oxygen supply to the vital organs in the body. However, the maximal heart rate achievable during exercise is heavily influenced by age. Most laboratories worldwide use a simple equation to predict the maximum heart rate: Maximum predicted heart rate $=220$ - age in years.

Traditionally, sufficient effort during treadmill ECG stress testing is defined as achieving $85 \%$ of the age-predicted maximal heart rate. However, some studies have reported a significant variability in the maximum predicted heart rate among people of identical ages; ${ }^{(5,6)}$ hence, this may not be used in isolation as a termination criterion. In addition to the evaluation of CAD, the treadmill ECG stress test is also often used to evaluate for chronotropic incompetency, in which the patient is unable to mount an adequate heart rate response to exercise, leading to symptoms such as effort-related dyspnoea and lethargy.

Table II. Characteristics of each stage of the Bruce protocol.

\begin{tabular}{lcccc}
\hline Stage & $\begin{array}{c}\text { Time } \\
(\mathbf{m i n})\end{array}$ & $\begin{array}{c}\text { Speed } \\
(\mathbf{k m} / \mathbf{h r})\end{array}$ & $\begin{array}{c}\text { Gradient } \\
\mathbf{( \% )}\end{array}$ & $\begin{array}{c}\text { Metabolic equivalents } \\
\text { of tasks }\end{array}$ \\
\hline 1 & 3 & 2.7 & 10 & 5 \\
2 & 6 & 4.0 & 12 & 7 \\
3 & 9 & 5.4 & 14 & 10 \\
4 & 12 & 6.7 & 16 & 13 \\
5 & 15 & 8.0 & 18 & 15 \\
6 & 18 & 8.8 & 20 & 18 \\
7 & 21 & 9.6 & 22 & 20 \\
\hline
\end{tabular}

\section{Blood pressure response}

The increase in sympathetic tone and vasodilation during exercise generally causes a rise in blood pressure. A drop in systolic blood pressure $>10 \mathrm{mmHg}$ is an indication to terminate the treadmill ECG stress tests, as it could be due to myocardial ischaemia leading to left ventricular dysfunction. This often signifies severe multi-vessel CAD, especially if there are accompanying symptoms and/or ST changes on the ECG. An exaggerated blood pressure response, defined as a peak systolic blood pressure of $>210 \mathrm{mmHg}$ in men and > $190 \mathrm{mmHg}$ in women according to the Framingham criteria, ${ }^{(7)}$ was shown to predict the risk of hypertension in normotensive individuals. ${ }^{(8)}$

\section{Indications for termination of the treadmill ECG stress test} In addition to achieving $85 \%$ of the age-predicted maximal heart rate, other indications for the termination of a treadmill ECG stress test are shown in Table III.

\section{Interpretation of the treadmill ECG stress test}

ST-segment changes indicative of obstructive CAD

A treadmill ECG stress test is considered abnormal when there is a horizontal or down-sloping ST-segment depression $\geq 1 \mathrm{~mm}$ at $60-80 \mathrm{~ms}$ after the J point. ${ }^{(9)}$ Exercise ECGs with up-sloping ST-segment depressions are typically reported as an 'equivocal' test. In general, the occurrence of horizontal or down-sloping ST-segment depression at a lower workload (calculated in METs) or heart rate indicates a worse prognosis and higher likelihood of multi-vessel disease. The duration of ST-segment depression is also important, as prolonged recovery after peak

Table III. Indications for termination of a treadmill ECG stress test. ${ }^{(9)}$

\begin{tabular}{|c|c|}
\hline Absolute indications & Relative indications \\
\hline $\begin{array}{l}\text { - ST-segment elevation of }>1 \mathrm{~mm} \text { in leads without pre-existing } \\
\mathrm{Q} \text { waves due to a prior myocardial infarction, other than aVR, } \\
\text { aVL and V1 } \\
\text { - Development of symptoms: moderate to severe angina, } \\
\text { dizziness, near-syncope } \\
\text { - Sustained ventricular tachycardia or other arrhythmia, including } \\
\text { second or third degree heart block that interferes with } \\
\text { maintenance of cardiac output during exercise } \\
\text { - Drop in systolic blood pressure }>10 \mathrm{mmHg} \text { despite an increased } \\
\text { workload, accompanied by other evidence of ischaemia } \\
\text { - Signs of poor perfusion (cyanosis, pallor) } \\
\text { - Neurological symptoms } \\
\text { - Individual's request to stop } \\
\text { - Technical difficulty in monitoring the ECG or systolic blood pressure }\end{array}$ & $\begin{array}{l}\text { - ST-segment depression (horizontal or down-sloping of }>2 \mathrm{~mm} \text { at } \\
60-80 \mathrm{~ms} \text { after the } \mathrm{J} \text { point in patient with suspected ischaemia } \\
\text { - Drop in systolic blood pressure }>10 \mathrm{mmHg} \text { despite an increased } \\
\text { workload without any other evidence of ischaemia } \\
\text { - Increasing chest pain, fatigue, dyspnoea, wheezing, leg cramps } \\
\text { or claudication } \\
\text { - Other tachyarrhythmia or bradyarrhythmia that may potentially } \\
\text { interfere with haemodynamic instability (e.g. supraventricular } \\
\text { tachycardia, multifocal ventricular ectopics) } \\
\text { - Exaggerated hypertensive response defined as systolic blood } \\
\text { pressure }>250 \mathrm{mmHg} \text { or diastolic blood pressure }>115 \mathrm{mmHg} \\
\text { - Development of bundle branch block that cannot be immediately } \\
\text { be distinguished from ventricular tachycardia }\end{array}$ \\
\hline
\end{tabular}

Table IV. Main contraindications for exercise stress test.

\begin{tabular}{ll}
\hline Absolute contraindication & Relative contraindication \\
\hline - Acute myocardial infarction or unstable angina & - Known obstructive left main coronary artery stenosis \\
- Symptomatic severe aortic stenosis & - Moderate to severe aortic stenosis \\
- Cardiac arrhythmia with haemodynamic instability & - Cardiac arrhythmia with rapid ventricular rates, advanced or complete \\
- Active endocarditis & heart blocks \\
- Decompensated heart failure & - Hypertrophic obstructive cardiomyopathy \\
- Acute myocarditis or pericarditis & - Recent stroke or transient ischaemic attack \\
- Acute pulmonary embolism, pulmonary infarction or & - Poorly controlled hypertension (resting blood pressure > 200/110 mmHg) \\
deep vein thrombosis & - Untreated medical conditions, such as anaemia and thyrotoxicosis \\
\hline
\end{tabular}


stress is consistent with a positive treadmill ECG stress test. Another finding that is highly indicative of significant CAD is the occurrence of ST-segment elevation $>1 \mathrm{~mm}$ (often suggesting transmural ischaemia); these patients are frequently referred urgently for coronary angiography.

In Case 1, the patient had an abnormal treadmill ECG stress test with mild horizontal and down-sloping ST depression in the inferior-lateral leads at Stage 2 (low workload) of the Bruce protocol. In addition to being electrically positive, the test was also subjectively positive, with the patient reporting chest pain with exercise stress. These are highly suggestive of obstructive $\mathrm{CAD}$, as proven in the subsequent coronary angiogram. Case 2 illustrated the use of the treadmill ECG stress test in a patient with a previous history of anterior myocardial infarction; the ST-segment depressions were seen not only at maximal heart rate, but also during the recovery period, indicating significant ischaemia. While ST-segment depression in the inferolateral leads during the treadmill ECG stress test indicates ischaemia from obstructive CAD, we cannot identify the diseased coronary artery from the ST-segment depression alone. However, as shown in Case 3, the ST-segment elevation during the treadmill ECG stress test can be used to identify the culprit coronary artery (where the stenosis causing ischaemia was located), which in this case was the RCA. Other possible causes of ST-segment elevation

ABSTRACT The treadmill electrocardiogram (ECG) stress test is widely used to screen for obstructive coronary artery disease (CAD). The presence of STsegment changes, either depression or elevation, on the ECG during the treadmill test often suggests presence of $C A D$ and warrants further management. We herein present three cases, with evidence of ischaemia on the treadmill ECG stress test. In addition, we discuss the use of the treadmill ECG stress test, including its indications, contraindications, reasons for termination and interpretation of the ST-segment changes, heart rate, as well as blood pressure responses to exercise. during the treadmill ECG stress test are coronary artery spasm and the presence of existing $\mathrm{Q}$ waves due to previous myocardial infarction.

In conclusion, the treadmill stress ECG test is a reliable screening test for obstructive CAD, especially in patients with an intermediate pretest probability. It is readily available in many centres in Singapore.

\section{REFERENCES}

1. Ministry of Health, Singapore. Singapore Health Facts: Principle Causes of Death [online]. Available at: https://www.moh.gov.sg/content/moh_web/ home/statistics/Health_Facts_Singapore/Principal_Causes_of_Death.html. Accessed May 1, 2016.

2. Gibbons RJ, Balady GJ, Beasley JW, et al. ACC/AHA guidelines for exercise testing: executive summary. A report of the American College of Cardiology/American Heart Association Task Force on Practice Guidelines (Committee on Exercise Testing). Circulation 1997; 96:345-54.

3. Gibbons RJ, Balady GJ, Bricker JT, et al; American College of Cardiology/ American Heart Association Task Force on Practice Guidelines. Committee to Update the 1997 Exercise Testing Guidelines. ACC/AHA 2002 guideline update for exercise testing: summary article. A report of the American College of Cardiology/American Heart Association Task Force on Practice Guidelines (Committee to Update the 1997 Exercise Testing Guidelines). J Am Coll Cardiol 2002; 40:1531-40.

4. Ministry of Health, Singapore. Screening for Cardiovascular Disease and Risk Factors. MOH Clinical Practice Guidelines 1/2011. Singapore: Ministry of Health, 2011.

5. Pinkstaff S, Peberdy MA, Kontos MC, Finucane S, Arena R. Quantifying exertion level during exercise stress testing using percentage of agepredicted maximal heart rate, rate pressure product, and perceived exertion. Mayo Clin Proc 2010; 85:1095-100.

6. Jain M, Nkonde C, Lin BA, Walker A, Wackers FJ. 85\% of maximal agepredicted heart rate is not a valid endpoint for exercise treadmill testing. J Nucl Cardiol 2011; 18:1026-35.

7. Lauer MS, Levy D, Anderson KM, Plehn JF. Is there a relationship between exercise systolic blood pressure response and left ventricular mass? The Framingham Heart Study. Ann Intern Med 1992; 116:203-10.

8. Singh JP, Larson MG, Manolio TA, et al. Blood pressure response during treadmill testing as a risk factor for new-onset hypertension. The Framingham heart study. Circulation 1999; 99:1831-6.

9. Fletcher GF, Ades PA, Kligfield P, et al; American Heart Association Exercise, Cardiac Rehabilitation, and Prevention Committee of the Council on Clinical Cardiology, Council on Nutrition, Physical Activity and Metabolism, Council on Cardiovascular and Stroke Nursing, and Council on Epidemiology and Prevention. Exercise standards for testing and training: a scientific statement from the American Heart Association. Circulation 2013; 128:873-934. 


\section{SINGAPORE MEDICAL COUNCIL CATEGORY 3B CME PROGRAMME} (Code SMJ 201607A)

Question 1. The following statement(s) is/are true:

(a) Ischaemic heart disease was the third leading cause of death in Singapore in 2014.

(b) The treadmill electrocardiogram (ECG) stress test is only used for diagnosis of obstructive coronary artery disease.

(c) An increase in blood pressure and decrease in heart rate is the body's normal physiological response to exercise.

(d) The target maximal heart rate is dependent on the individual's age.

Question 2. According to the American Heart Association's recommendation, the treadmill ECG stress test is an appropriate test for the following patient(s):

(a) A 30-year-old woman with pleuritic chest pain.

(b) A 40-year-old man with angina pectoris.

(c) An asymptomatic 55-year-old woman.

(d) A 62-year-old man with atypical chest pain.

Question 3. The treadmill ECG stress test is contraindicated in:

(a) Severe aortic stenosis.

(b) Severe mitral regurgitation.

(c) Atrial fibrillation with rapid ventricular rate.

(d) Chronic heart failure.

Question 4. The treadmill ECG stress test must be terminated in the following situation(s):

(a) 1-mm ST-depression in the inferior leads.

(b) A drop in systolic blood pressure of more than $10 \mathrm{mmHg}$ despite an increased workload.

(c) The development of complete heart block during the test.

(d) The development of mild chest discomfort during the test.

Question 5. In a subject with normal baseline ECG, the treadmill ECG stress test is considered electrically positive in the following:

(a) ECG showing 0.5-mm up-sloping ST-segment depression at peak heart rate with absence of symptoms.

(b) ECG showing 2-mm ST-segment elevation at 70\% of target heart rate.

(c) ECG showing 1-mm up-sloping ST-segment depression at J point at $86 \%$ of the target heart rate.

(d) ECG showing 1-mm down-sloping ST-segment depression at $80 \mathrm{~ms}$ after the J point at $85 \%$ of the target heart rate.

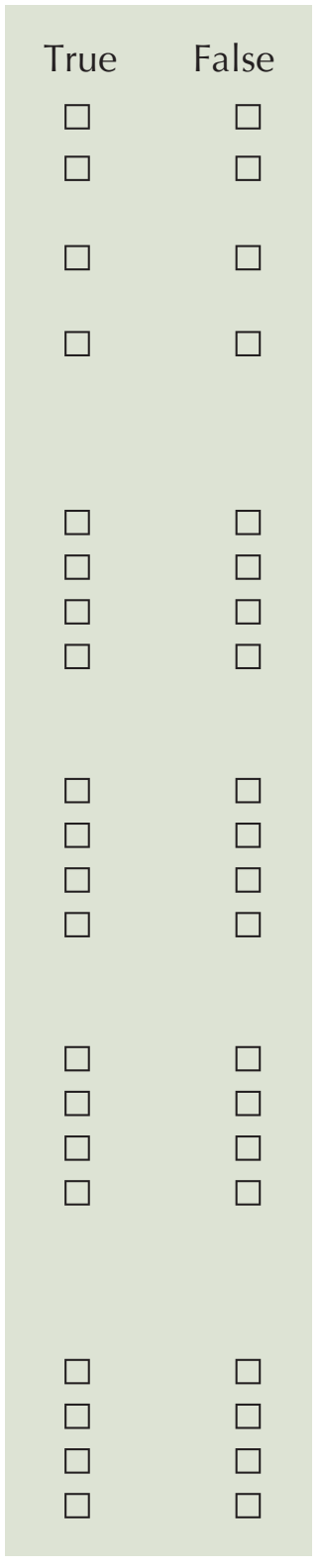

\footnotetext{
Doctor's particulars:

Name in full

MCR number

Specialty:

Email address

SUBMISSION INSTRUCTIONS:

(1) Visit the SMJ website: http://www.smj.org.sg/current-issue and select the appropriate set of questions. (2) Provide your name, email address and MCR number. (3) Select your answers and click "Submit".

RESULTS:

(1) Answers will be published online in the SMJ September 2016 issue. (2) The MCR numbers of successful candidates will be posted online at the SMJ website by 2 September 2016. (3) Passing mark is $60 \%$. No mark will be deducted for incorrect answers. (4) The SMJ editorial office will submit the list of successful candidates to the Singapore Medical Council. (5) One CME point is awarded for successful candidates.

Deadline for submission: (July 2016 SMJ 3B CME programme): 12 noon, 26 August 2016.
} 